\title{
Acessibilidade em Websites de Instituições Escolares com foco no Atendimento aos Usuários Surdos
}

\author{
Guilherme José Basso, Soraia Silva Prietch \\ Curso de Licenciatura em Informática - Universidade Federal de Mato Grosso \\ Avenidas dos Estudantes, no 5055 - CEP 78735-901 - Rondonópolis - MT - Brasil \\ guilhermebasso2@hotmail.com, soraiadufmt.br
}

\begin{abstract}
The goal of this study was to evaluate two websites of educational institutions to check accessibility features for people who are Deaf or Hard of Hearing (D/HH), focusing on the use of automatic evaluators. As a result, we did not find elements of accessibility for people who are $\mathrm{D} / \mathrm{HH}$ and these websites were not in accordance with accessibility standards. Moreover, it was possible to make a comparison between automatic evaluators, considering their different ways of reporting issues.
\end{abstract}

Resumo. O objetivo desse trabalho foi avaliar dois websites de instituições escolares para verificar características de acessibilidade para pessoas Surdas ou com Deficiência Auditiva (S/DA), com enfoque no uso de avaliadores automáticos disponíveis. Obteve-se como resultado que, nos websites pesquisados, não foram encontrados elementos de acessibilidade para pessoas S/DA e que estes websites não estavam de acordo com as normas de acessibilidade. Além disso, foi possível efetuar um comparativo entre as diferentes formas de apresentação de resultados emitidos pelos avaliadores automáticos.

\section{Introdução}

O problema que motivou o desenvolvimento deste trabalho foi a falta de acessibilidade para usuários Surdos ou Deficientes Auditivos (S/DA) em websites escolares. Segundo Schelp (2009, p. 3037), "[...] o desempenho acadêmico e social esperado da criança surda só pode ser alcançado se no espaço escolar for contemplada sua condição lingüística e cultural e, portanto, se a língua de sinais fizer-se presente". Quando é mencionado espaço escolar este também se refere ao espaço digital proporcionado pela escola, ambiente em que são disponibilizadas informações relevantes aos estudantes, pais e professores. De acordo com Ribeiro, Fonseca e Santos (2010, p. 1317) "Mesmo que a tarefa [objetivo a ser atingido] não seja escolar [educacional], o uso do computador para fins de sobrevivência no trabalho ou nas relações sociais é de suma importância para algumas comunidades". Nesse contexto, os usuários S/DA podem ser estudantes ou potenciais estudantes de alguma escola, podem ser pais S/DA de estudantes ouvintes, podem ser professores $\mathrm{S} / \mathrm{DA}$, dentre outros tipos de usuários.

Considerando o problema, é relevante citar a Lei $\mathrm{n}^{\circ} 10.436$ [Brasil 2002], o Decreto $n^{\circ} 5.296$ [Brasil 2004] e a Lei $n^{\circ} 13.146$ [Brasil 2015], os quais reforçam a importância tanto das Tecnologias de Comunicação e Informação (TIC) como um dos fatores de promoção de acessibilidade, a obrigatoriedade de instituições de garantir o 
acesso às atividades escolares de forma igualitária, e a regulamentação e oferta da Língua Brasileira de Sinais (Libras), como primeira língua das pessoas surdas brasileiras.

No que se refere aos usuários S/DA, Cavender e Ladner (2008) evidenciam características específicas das pessoas $\mathrm{S} / \mathrm{DA}$ ao utilizar sistemas computacionais, mencionando principalmente a questão da língua de sinais (visual-gestual) e a forma como o pensamento se desenvolve em função desse aspecto. Um aspecto relevante é que a grande parte da comunidade surda brasileira, especialmente as pessoas que nasceram surdas ou ficaram surdas a partir da infância, preferem se comunicar e receber informações em Libras. Além disso, nem todas as pessoas surdas conseguem realizar leitura labial [WebAIM/AD 2013] e, mesmo as que conseguem, essas compreendem 40$60 \%$ do que foi dito; e, segundo Medeiros, Elia e Santos (2013) um número expressivo de pessoas surdas possui dificuldades com a leitura e escrita de textos.

A justificativa de realização deste trabalho, portanto, consiste em abordar o problema apresentado, destacando as características de uso de websites por pessoas surdas, identificadas em pesquisas prévias, a fim de apresentar resultados com utilidade prática. Considerando o exposto, alguns questionamentos surgiram, os quais pretendeuse responder ao longo deste estudo: (1) Quais são os elementos de acessibilidade recomendados na literatura para surdos?; (2) Quais as vantagens e desvantagens de se utilizar um avaliador automático de acessibilidade de websites?; (3) Os websites avaliados contam com elementos de acessibilidade para usuários surdos?

Neste contexto, o objetivo geral desse trabalho é avaliar dois websites de instituições escolares para verificar características de acessibilidade para pessoas S/DA, com enfoque no uso de avaliadores automáticos confrontando esses resultados com os elementos de acessibilidade validados na literatura para o público-alvo em questão.

O presente artigo está organizado nas seguintes seções: a Seção 2 descreve os materiais e métodos; a Seção 3 apresenta um breve embasamento teórico; a Seção 4 traz o relato da avaliação realizada nos websites escolares; a Seção 5 dispõe os resultados e a discussão; e, a Seção 6 faz o fechamento com as conclusões.

\section{Materiais e Métodos}

Para atingir o objetivo da pesquisa, cinco etapas foram seguidas. A Etapa 1 consistiu na revisão de literatura, para buscar trabalhos correlatos e proporcionar embasamento teórico para dar suporte às etapas posteriores de pesquisa.

Na Etapa 2 preocupou-se em efetuar o levantamento, em 2014, dos avaliadores automáticos de acessibilidade. A relação de 119 avaliadores automáticos ${ }^{1}$ de acessibilidade, disponíveis na ocasião, no website do W3C foi analisada conforme os seguintes critérios: (Critério 1) Excluir aqueles que não estiverem de acordo com as normas atuais de acessibilidade digital (WCAG 2.0); (Critério 2) Excluir aqueles que são avaliadores de linguagens de marcação ou folha de estilos; (Critério 3) Excluir aqueles avaliadores que não estão mais disponíveis para uso; (Critério 4) Excluir os avaliadores que não constam em língua inglesa, portuguesa ou espanhola; (Critério 5) Excluir avaliadores de contraste de cores, leitores de tela, epilepsia, ou que necessita de

\footnotetext{
${ }^{1}$ www.w3.org/WAI/RC/tools/complete.
} 
instalação para uso ou pago, ou guias; que não são foco desta pesquisa; (Critério 6) Avaliadores que possuem várias versões, foi considerada apenas uma.

$\mathrm{Na}$ Etapa 3 definiu-se quais websites de escolas e quais páginas dos websites seriam avaliados. Os websites das seguintes instituições escolares foram escolhidos como objetos de estudo: Escola Estadual La Salle, e Centro Educacional Khalil Zaher - ambas localizadas na cidade de Rondonópolis-MT. A escolha destas instituições justificou-se pelo fato de que: a primeira é uma escola estadual de referência na cidade e que possui um website, diferente das demais escolas que contam com blogs; e, a segunda é uma escola particular de ensino fundamental e médio que possui sala para o Atendimento Educacional Especializado. Apenas uma página de cada website foi escolhida para avaliação. No caso da escola La Salle foi a página Home (Início ou Index) e, no caso, da escola Khalil foi a página Home do ensino fundamental. Essa decisão foi tomada para reduzir o universo de observação, devido às múltiplas possibilidades de navegação em profundidade que um website pode oferecer.

A Etapa 4 consistiu na efetiva avaliação dos websites. Nesta etapa, interessava-se em descobrir que tipos de falhas de acessibilidade os avaliadores automáticos conseguem identificar e avaliar as páginas dos websites escolares selecionados. Para a avaliação, foram realizados os seguintes passos para cada escola: (1) Acesso ao website do avaliador automático de acessibilidade; (2) Inserção do endereço eletrônico do website da escola no campo indicado para avaliação no website do avaliador automático; (3) Se as opções de escolha da norma ou dos níveis de conformidade de uma norma para avaliação estivéssem disponíveis, uma avaliação para cada opção foi realizada (exemplo, norma WCAG 2.0: Níveis A, AA e AAA); senão, foi inspecionada de acordo com as normas padrão (default) do avaliador automático; e, (4) Após a exibição do resultado, cada tela foi capturada para compor um relatório completo por página das escolas.

Por fim, na Etapa 5, foi realizada uma validação humana, com a intenção de complementar os resultados fornecidos pelos avaliadores automáticos de acessibilidade, para se certificar de que os elementos de acessibilidade realmente foram evidenciados.

\section{Acessibilidade digital para Pessoas Surdas ou com Deficiência Auditiva}

Conforme Silva et al. (2005, p. 2469),

Um dos fatores que mais prejudicam a inclusão social de pessoas com necessidades especiais [Pessoas com Deficiência, PcD] é seu acesso limitado a informação. Uma parcela significativa da população mundial tem algum tipo de deficiência que reduz efetivamente sua habilidade para usar novas tecnologias, tais como computadores e a Internet. Sendo assim, é de grande importância tornar a informação eletrônica acessível, afim de ajudar as pessoas com necessidades especiais $[\mathrm{PcD}]$ a se reintegrarem socialmente.

A acessibilidade é definida pela ISO/IEC 25010 (2011, p. 21) como "o grau em que um produto ou sistema pode ser usado por pessoas com uma ampla variedade de características e capacidades para atingir um objetivo específico em um contexto de uso específico". A referida norma ISO, ainda, torna clara a relação entre usabilidade e acessibilidade, visto que sistemas sem usabilidade se tornam sistemas inacessíveis por grande parte dos usuários. Ao mencionar "pessoas com uma ampla variedade de características e capacidades", esta não compreende somente pessoas com deficiência, 
V Congresso Brasileiro de Informática na Educação (CBIE 2016)

Anais do XXII Workshop de Informática na Escola (WIE 2016)

mas pessoas que sofreram algum tipo de acidente e estão temporariamente incapacitadas, pessoas idosas que tiveram suas habilidades reduzidas, crianças ou adultos que ainda não são alfabetizados, dentre outras.

A Iniciativa de Acessibilidade Web (Web Accessibility Initiative, WAI) é uma ramificação da Consórcio World Wide Web (World Wide Web Consortium, W3C), a qual é responsável por propor "estratégias, diretrizes e recursos para tornar a web acessível para pessoas com deficiência" (WAI/W3C, s/d, tradução nossa). Dentre os documentos da WAI/W3C, somente as diretrizes WCAG 2.0 são abordadas neste artigo devido ao foco de interesse da presente pesquisa.

A WCAG refere-se às recomendações de acessibilidade para conteúdos da Web, a qual possui diversas camadas de orientações para que projetistas e outros profissionais sejam capazes de desenvolver ou identificar falhas e acertos de acessibilidade em websites. De modo geral, estas orientações compreendem os Princípios, as Diretrizes, os Critérios de Sucesso, e as Técnicas do tipo Necessária e do tipo Sugerida. Os Princípios são quatro: Perceptível, Operável, Compreensível, e Robusto. As Diretrizes (WCAG/W3C 2.0, 2014) estão associadas diretamente aos Princípios e são desdobradas em categorias enumeradas, que por sua vez são desdobradas nos critérios de sucesso (por exemplo, a Diretriz 4.1 se desdobra nos Critérios de sucesso 4.1.1 e 4.1.2), sendo que as subcategorias são classificadas por nível de conformidade (A, AA ou AAA). Os níveis de conformidade (WCAG/W3C 2.0, 2014) são responsáveis por classificar as diretrizes de modo que os projetistas possam atingir critérios de sucesso em termos de promoção da acessibilidade em websites; sendo que o nível A é mais significativo.

Conforme Flor, Vanzin e Ulbricht (2013, p. 165-166), na WCAG 2.0, "Dos 61 critérios de sucesso, apenas seis foram relacionados à surdez [...]”, a citar: (1.2.1) Apenas áudio e apenas vídeo (Pré-gravado); (1.2.2) Legendas (Pré-gravadas); (1.2.4) Legendas (Ao vivo); (1.2.6) Linguagem de sinais (Pré-gravada); (1.2.8) Mídia alternativa (Pré-gravada); e, (1.2.9) Apenas áudio (Ao vivo). É válido ressaltar que o áudio também é uma alternativa de acessibilidade, por que existem usuários que possuem surdez unilateral ou usuários que possuem deficiência auditiva leve ou moderada.

Além da WCAG 2.0, que é uma norma internacional, o Brasil também possui uma norma própria para orientar a promoção da acessibilidade em governo eletrônico, denominada e-MAG (Modelo de Acessibilidade em Governo Eletrônico, www.governoeletronico.gov.br/acoes-e-projetos/e-MAG), que no momento da realização da presente pesquisa encontrava-se na versão 3.0. $\mathrm{O}$ primeiro princípio recomenda o uso de Padrões Web (Web Standards) da W3C; o segundo princípio sugere seguir as diretrizes e recomendações de acessibilidade da norma WCAG 2.0; e, o terceiro princípio se refere ao uso de avaliadores automáticos de acessibilidade.

Considerando a diversidade de comunicação da pessoa S/DA, Corradi (2007, p. 124-126) investigou 13 (treze) Elementos de Acessibilidade Digital, para Pessoas Surdas: (1) Presença de legendas (closed caption) em português para vídeos; (2) Presença da Língua de Sinais/ LIBRAS em ambientes digitais; (3) Apresentação de conteúdo em imagens, fotografias e sons disponíveis também em formato de texto em português; (4) Apresentação de textos em português disponíveis também em vídeos dinâmicos em LIBRAS; (5) Vídeos em LIBRAS com legendas em português; (6) Apresentação de conteúdos digitais em diferentes formatos (texto, imagem, vídeo e som) 
e em hipermídia; (7) Diferenciação de cores entre os conteúdos e links consultados; (8) Alternativas de mudanças de cor, tamanho da fonte, tamanho da tela, e ajustes de som; (9) Presença do SignWriting (escrita da Língua de Sinais) em ambientes digitais; (10) Controle do usuário sobre as apresentações das informações (voltar, adiantar, parar, começar); (11) Disponibilizar dicionários digitais da LIBRAS para consulta; (12) Mecanismos de ajuda que auxiliem o usuário diante de suas dificuldades de navegação e fornecimento de respostas (feedback) às suas dúvidas por meio digital (e-mail); e, (13) Disponibilizar o Player Rybená (ou outro) para acessar o conteúdo informacional digital.

\section{Avaliação dos Websites Escolares}

Seguindo as etapas da metodologia de pesquisa, inicialmente ocorreu a definição dos avaliadores automáticos de acessibilidade que seriam utilizados na pesquisa. Como resultado dessa definição obteve-se os seguintes: A-Checker, TAW Online, AccessMonitor e DaSilva. Apenas uma página de cada website escolar foi escolhida para efetuar a avaliação automática de acessibilidade. No caso da escola La Salle foi a página Home e, no caso, da escola Khalil foi a página Home do ensino fundamental. Cada página foi avaliada pelos quatro avaliadores automáticos. As capturas de tela dos relatórios emitidos na íntegra pelos avaliadores automáticos foram compilados em um único arquivo por escola, o qual consta disponível em disco virtual (http://goo.gl/cKW6G6). Na sequência, constam os resultados resumidos das avaliações.

\subsection{Avaliações automáticas de uma página de cada website das escolas}

Analisando os resultados apontados pelos avaliadores automáticos, com relação à página Home do website da escola La Salle, interessava-se em descobrir quais foram os erros ou avisos nos critérios de sucesso mais frequentes. Isso porque considerou-se que se todos os avaliadores automáticos apresentaram erros ou avisos para os mesmos critérios de sucesso, estes precisam de mais atenção. Os critérios de sucesso considerados mais críticos são aqueles 04 apontados pelos quatro avaliadores automáticos (1.1.1, 1.3.1, 3.1.1, 3.3.2), sendo todos referentes ao Nível de conformidade A (mais significativos). É válido notar que nenhum avaliador automático evidenciou erro ou aviso nos seis critérios de sucesso para atendimento de conteúdos Web aos usuários S/DA, frisados por Flor, Vanzin e Ulbricht (2013). Além disso, o avaliador AccessMonitor classificou a página com um índice (nota) de 4.6, o que foi considerado abaixo da média, visto que esse índice utiliza a escala de 0 a 10.

A mesma preocupação apontada na avaliação da página da Escola La Salle, também foi explorada para a página da Escola Khalil; em conhecer quais foram os critérios de sucesso mais frequentes apontados pelos avaliadores automáticos. Como resultado, os critérios de sucesso mais críticos foram 04 apontados pelos quatro avaliadores automáticos $(1.1 .1,2.4 .4,3.1 .1,4.1 .1)$, sendo todos referentes ao Nível de conformidade A (mais significativos). Assim como foi verificado na página da Escola La Salle, nenhum avaliador automático evidenciou erro ou aviso nos seis critérios de sucesso para atendimento de conteúdos Web aos usuários S/DA, frisados por Flor, Vanzin e Ulbricht (2013). No entanto, neste caso, o avaliador TAW classificou tais critérios de sucesso como itens não verificados. Além disso, o avaliador AccessMonitor classificou a página com um índice (nota) de 3.9, sendo esse mais baixo do que o índice atribuído pelo avaliador para a página da Escola La Salle (4.6). 
Durante a avaliação automática, os avaliadores retornaram como resultado itens não verificados. No relatório de avaliação, não constou uma explicação do motivo pelo qual estes itens não foram verificados; assumiu-se que o avaliador não tinha recursos suficientes disponíveis para efetivar a avaliação do item, visto que dez itens não foram citados como erro, aviso ou item não verificado.

\subsection{Validação humana de uma página de cada website das escolas em questão}

Devido ao resultado das avaliações automáticas, decidiu-se efetuar a validação humana de acessibilidade das mesmas páginas, a fim de averiguar os critérios de sucesso de interesse, em cujos relatórios ou não apontaram erros/avisos ou identificaram como itens não verificados. Para esta finalidade, elaborou-se uma checklist, com as seguintes opções de respostas: $\mathrm{AC}=$ Atende Completamente $(100 \%)$; $\mathrm{AP}=$ Atende Parcialmente; $\mathrm{NA}=$ Não Atende. A opção de resposta AP subdivide-se em três tipos: $75 \%=$ atende em grande parte, $50 \%=$ atende de forma mediana, ou $25 \%=$ atende precariamente. No Quadro 1, é apresentado a checklist utilizada e o resultado da validação humana com relação aos elementos de acessibilidade para pessoas S/DA nas páginas dos websites da Escola La Salle e da Escola Khalil Zaher.

Tabela 1. Checklist de validação humana

\begin{tabular}{|c|c|c|c|c|c|c|c|c|c|c|}
\hline \multirow{3}{*}{ WCAG 2.0 para usuários surdos } & \multicolumn{5}{|c|}{ Escola La Salle } & \multicolumn{5}{|c|}{ Escola Khalil Zaher } \\
\hline & \multirow{2}{*}{ AC } & \multicolumn{3}{|c|}{ AP (\%) } & \multirow{2}{*}{ NA } & \multirow{2}{*}{$\mathbf{A C}$} & \multicolumn{3}{|c|}{ AP (\%) } & \multirow{2}{*}{ NA } \\
\hline & & 75 & 50 & 25 & & & 75 & 50 & 25 & \\
\hline 1.2.1 Apenas Audio e apenas Vídeo (Pré-gravado) & & & & & $\mathrm{X}$ & & & & & $\mathrm{X}$ \\
\hline 1.2.2 Legendas (Pré-gravadas) & & & & & $\mathrm{X}$ & & & & & $\mathrm{X}$ \\
\hline 1.2.4 Legendas (Ao Vivo) & & & & & $\mathrm{X}$ & & & & & $\mathrm{X}$ \\
\hline 1.2.6 Linguagem de sinais (Pré-gravada) & & & & & $\mathrm{X}$ & & & & & $\mathrm{X}$ \\
\hline 1.2.8 Mídia Alternativa (Pré-gravada) & & & & $\mathrm{X}$ & & & & & $\mathrm{X}$ & \\
\hline 1.2.9 Apenas áudio (Ao vivo) & & & & & $\mathrm{X}$ & & & & & $\mathrm{X}$ \\
\hline
\end{tabular}

Fonte: Adaptado de Flor, Vanzin e Ulbricht (2013, p. 165).

É possível observar, pela checklist usada durante a validação humana, que 05 dos 06 critérios de sucesso não atendem os usuários S/DA ao navegar nas páginas Web avaliadas, uma vez que esses elementos de interface de promoção à acessibilidade não constam nas mesmas. Entretanto, alguns critérios de sucesso não são verificados. Isso não significa que os elementos de acessibilidade estão disponíveis ou estão incorretos. Por exemplo, o critério de sucesso 1.2.2, que trata das legendas em materiais previamente gravados, não foi apontado com erro ou aviso, mas isso porque não constava nenhum material em vídeo ou áudio nos websites escolares avaliados. Ou seja, não houve falha nesse critério, mas isso não significa que os websites avaliados sejam acessíveis para usuários S/DA, afinal esse elemento de acessibilidade não está presente.

$\mathrm{O}$ único critério de sucesso atendido parcial e precariamente é o 1.2.8, referente à opção de mídia alternativa pré-gravada. Esse critério (1.2.8) foi marcado como AP 25\% por que nas páginas avaliadas (Home) constam algumas imagens que são explicadas em texto, visto que a comunicação das informações ainda está incompleta. Além disso, durante a validação foi possível navegar pelo website da escola La Salle para conhecer as demais páginas vinculadas ao mesmo. Com esta navegação se tornou claro que a metade dos hiperlinks constantes no website levam o usuário para páginas externas sem aviso prévio, o sistema de busca localiza a palavra-chave em todos os websites da rede de escolas e não somente no website da escola, e a última atualização foi realizada em 2011. 
V Congresso Brasileiro de Informática na Educação (CBIE 2016)

Anais do XXII Workshop de Informática na Escola (WIE 2016)

Considerou-se a mídia alternativa pré-gravada como sendo um elemento que atende parcialmente (vinte e cinco por cento). Isso por constar imagens e uma animação em que algumas destas são explicadas em texto. Entretanto, novamente deve-se frisar que as imagens com texto conferiam explicações insuficientes.

\section{Resultados e Discussão}

Após a validação humana, se tornaram claras as diferenças entre os websites escolares avaliados, sendo que o da Escola Khalil vem sendo atualizado com frequência e conta com diversas informações importantes que consideram diferentes tipos de potenciais usuários; sendo que tais evidências não foram visualizadas no website da Escola La Salle. No entanto, mesmo com esta vantagem para o website da escola Khalil, ambos websites falharam no atendimento dos elementos de acessibilidade de conteúdos web para usuários S/DA.

Além disso, ao comparar a forma com que cada avaliador (1: A-Checker, 2: AccessMonitor, 3: DaSilva, e 4: TAW) apresenta seus resultados, é possível observar que estes são mostrados de maneiras diferentes na tela. Neste sentido, no Quadro 2 são listados os pontos positivos e negativos dos avaliadores automáticos.

Tabela 2. Pontos positivos e negativos dos avaliadores automáticos

\begin{tabular}{|c|c|c|c|c|}
\hline Quesitos avaliados pelos autores desse artigo & \multicolumn{4}{|c|}{ Avaliadores } \\
\hline$\checkmark$ significa ponto positivo para o avaliador em questão & 1 & 2 & 3 & 4 \\
\hline $\begin{array}{l}\text { 1. Ao acessar a página do avaliador, o local onde se deve inserir o hiperlink do } \\
\text { website a ser avaliado e o acionamento do sistema para verificação são } \\
\text { intuitivos e fáceis de encontrar/ compreender }\end{array}$ & $\checkmark$ & $\checkmark$ & $\checkmark$ & $\checkmark$ \\
\hline 2. Para utilizar o avaliador não se faz necessário realizar registro / login & $\checkmark$ & $\checkmark$ & $\checkmark$ & $\checkmark$ \\
\hline $\begin{array}{l}\text { 3. Indicação do Nível de conformidade e do Critério de sucesso aos quais as } \\
\text { falhas (erros/avisos) encontradas estão relacionadas }\end{array}$ & $\checkmark$ & $\checkmark$ & $\checkmark$ & $\checkmark$ \\
\hline 4. Informação da linha do código em que a falha ocorre & $\checkmark$ & & $\checkmark$ & $\checkmark$ \\
\hline 5. Sugestão de como reparar a falha apontada & $\checkmark$ & $\checkmark$ & & \\
\hline $\begin{array}{l}\text { 6. Ao clicar no hiperlink do item checado, uma nova página é aberta contendo } \\
\text { seus detalhamentos, como: resultados esperados, exemplos de reparos, etc }\end{array}$ & $\checkmark$ & & & \\
\hline $\begin{array}{l}\text { 7. Possibilidade de exportar os resultados em forma de relatório (completo ou } \\
\text { parcial) em variados tipos de extensões de arquivos }\end{array}$ & $\checkmark$ & & & \\
\hline $\begin{array}{l}\text { 8. A partir da própria página de resultados é possível efetuar nova avaliação } \\
\text { sem precisar retornar à página Home do avaliador }\end{array}$ & $\checkmark$ & & $\checkmark$ & \\
\hline 9. Opção de apresentação do resultado em outras línguas & $\checkmark$ & & & $\checkmark$ \\
\hline $\begin{array}{l}\text { 10. Possibilidade de avaliar automaticamente os três níveis de conformidade } \\
\text { WCAG 2.0, ou de associar diferentes normas para avaliar, simultaneamente }\end{array}$ & & $\checkmark$ & $\checkmark$ & \\
\hline $\begin{array}{l}\text { 11. Existência de elementos de acessibilidade no website como, por exemplo, } \\
\text { opções de contraste, alteração do tamanho da fonte, auxílios para leitores de } \\
\text { tela, conteúdo em LIBRAS, dentre outros }\end{array}$ & & & & \\
\hline 12. Atribuição de uma nota final (geral) da avaliação & & $\checkmark$ & & \\
\hline 13. Apresenta resumo dos resultados & & $\checkmark$ & & $\checkmark$ \\
\hline TOTAL DE PONTOS POSITIVOS = & 09 & 07 & 06 & 06 \\
\hline
\end{tabular}

Fonte própria.

A partir do Quadro 2 é possível perceber características positivas em todos os avaliadores, porém o A-Checker acumula mais pontos positivos do que os demais, sendo seguido pelo AccessMonitor, DaSilva e TAW online, respectivamente. Se existisse um avaliador que agregasse todos os pontos positivos simultaneamente encontrados nos quatro avaliadores seria uma ferramenta mais adequada e desejável. Um fator interessante, e de certa forma incoerente, a ser observado é que nenhum dos quatro avaliadores automáticos conta com elementos de acessibilidade em seus websites; ou 
seja, um projetista, ou uma pessoa interessada, com deficiência que desejasse efetuar a avaliação de websites talvez não conseguisse, ou demorasse para atingir o seu objetivo. Em suma, avaliadores de acessibilidade se mostraram inacessíveis.

Outro ponto importante a ser comentado com relação aos resultados apresentados pelos avaliadores é o tipo e a quantidade de falhas identificados pelos mesmos. No que se refere à escola pública estadual La Salle, os dados quantitativos relativos aos erros e avisos encontrados pelos avaliadores automáticos para os Princípios com maior quantidade de erros foram os Princípios 1 e 3, sendo evidenciados pelo AChecker, DaSilva e TAW online. Ainda com relação à escola La Salle, a Figura 1 mostra os quantitativos relativos aos erros (azul) e avisos (vermelho) encontrados pelos avaliadores automáticos para os Níveis de Conformidade A, AA, e AAA.

(a) $\mathrm{A}$

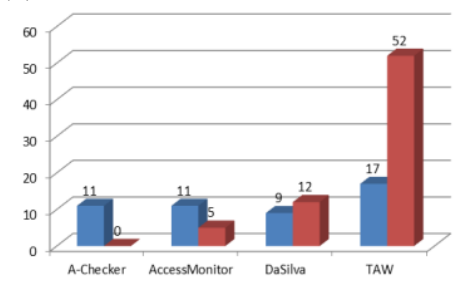

(b) AA

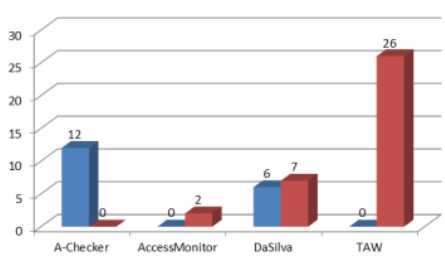

(c) AAA

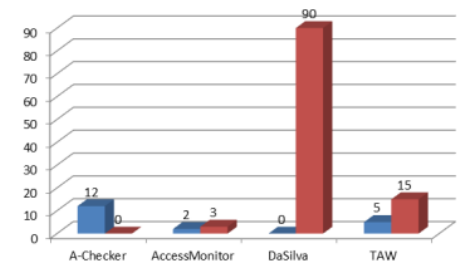

Figura 1 - Quantitativos relativos aos erros e avisos encontrados pelos avaliadores para os níveis de conformidade: escola La Salle

Os quantitativos dos níveis de conformidade da WCAG 2.0 são analisados aqui por avaliador automático, o que torna fácil perceber que em todos os níveis foram encontrados mais avisos do que erros, assim como ocorreu para os princípios. O avaliador TAW indicou o maior número de avisos para os níveis A e AA, e o DaSilva apontou o maior número de avisos para o nível AAA. A maior quantidade de erros evidenciados pelos avaliadores foi o nível A, e a menor para o nível AAA.

Em comparação com a página avaliada do website da escola La Salle, a página avaliada, do website da escola Khalil, apresenta número muito maior de erros e avisos, sendo que o maior número apresentado foi para o Princípio 3. Ainda com relação à escola Khalil, a Figura 2 mostra os quantitativos relativos aos erros (azul) e avisos (vermelho) encontrados pelos avaliadores automáticos para os Níveis de Conformidade.

(a) $\mathrm{A}$

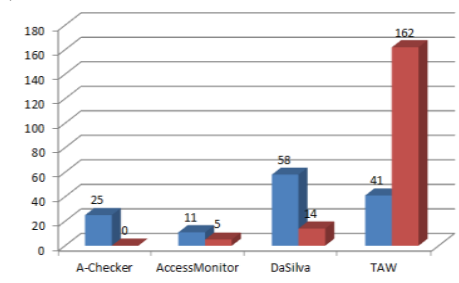

(b) AA

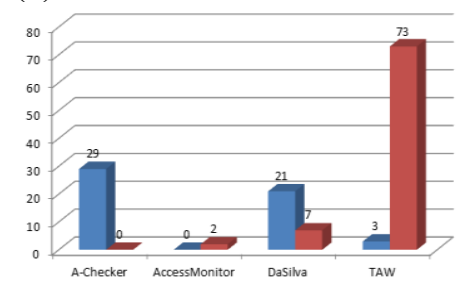

(c) AAA

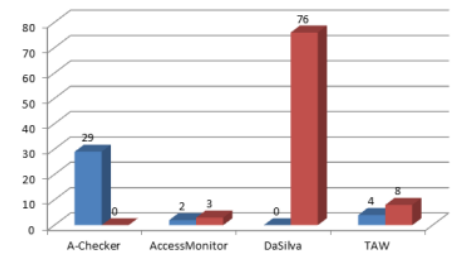

Figura 2. Quantitativos relativos aos erros e avisos encontrados pelos avaliadores automáticos para os níveis de conformidade: escola Khalil Zaher

Os quantitativos dos níveis de conformidade da WCAG 2.0 foram analisados também por avaliador automático, o que torna fácil perceber que em todos os níveis foram encontrados mais avisos do que erros, assim como ocorreu para os princípios. $\mathrm{O}$ avaliador TAW indicou o maior número de avisos para os níveis A (com 162) e AA 
(com 73), e o DaSilva apontou o maior número de avisos para o nível AAA (com 76). A maior quantidade de erros evidenciados pelos avaliadores foi o nível A, e a menor para o nível AAA, assim como ocorreu na avaliação da página do website da escola La Salle.

\section{Conclusão}

Respondendo a questões de pesquisa: Quais são os elementos de acessibilidade recomendados na literatura para surdos?, foi possível averiguar que os elementos de acessibilidade estão ligados diretamente aos modos de comunicação dos usuários surdos. Por exemplo, para o usuário que se comunica em Libras é interessante que o website disponibilize vídeos ou animações nessa língua. Neste aspecto, o trabalho de Corradi (2007) e de Martins (2012) relatam investigações específicas com o público-alvo de interesse neste trabalho e fornecem recomendações de elementos de acessibilidade.

Como resposta para a pergunta: Quais as vantagens e desvantagens de se utilizar um avaliador automático de acessibilidade de websites?, tem-se que alguns resultados dos avaliadores automáticos de acessibilidade indicaram quais os critérios de sucesso apresentaram erros ou avisos a serem corrigidos e também apontam a linha de comando em que determinado erro ou aviso ocorreu. Essa segunda parte dos resultados é mais voltada para desenvolvedores, não para usuários finais. Portanto, os relatórios emitidos são técnicos e muitas vezes difíceis de serem interpretados. Por outro lado, a facilidade de se inserir o endereço eletrônico em um campo e aguardar que a avaliação seja realizada automaticamente é algo prático e rápido, sendo que diversas correções e melhorias podem ser implementadas a partir do relatório do avaliador.

No que tange à questão: Os websites avaliados contam com elementos de acessibilidade para usuários surdos?, pode-se perceber que ainda existe falta de informação a respeito dos usuários S/DA com relação ao acesso a conteúdos web. Isso ocorre por que a maioria das pessoas acredita que todos os usuários S/DA são capazes de ler fluentemente quaisquer tipos de texto em língua escrita, e isso não é uma verdade, conforme Medeiros, Elia e Santos (2013). Neste aspecto, os websites escolares avaliados possuem basicamente textos, e imagens e animações sem descrições, e não contemplam alternativas como vídeos em Libras, que é a língua materna e oficial das pessoas surdas brasileiras. Verificou-se que além dos websites das instituições escolares avaliados não contemplarem de forma adequada os elementos de acessibilidade de conteúdos Web para usuários S/DA, ainda possuem diversas outras falhas de usabilidade e de arquitetura da informação que impedem o acesso a uma gama maior de outros potenciais usuários. A resposta é que os websites avaliados atendem precariamente potenciais usuários S/DA.

Como forma de continuidade deste trabalho, pretende-se apresentar os resultados às escolas, cujos websites foram avaliados, e buscar estabelecer diálogo com a Secretaria de Educação para verificar a possibilidade colaboração para o projeto e/ou avaliação da acessibilidade dos websites das escolas de Rondonópolis. Isso por que os websites escolares podem ser interfaces entre Instituições de Ensino e Sociedade, fornecendo informações, promovendo interlocução entre as demandas dos pais e dos bairros com as escolas, divulgando projetos e atividades realizadas por alunos e professores, disponibilizando instrumentos de avaliação, dentre outros. 
V Congresso Brasileiro de Informática na Educação (CBIE 2016)

Anais do XXII Workshop de Informática na Escola (WIE 2016)

\section{Referências}

Brasil. Lei $\mathrm{n}^{\circ}$. 10.436, de 24/04/2002. Dispõe sobre a Língua Brasileira de Sinais. Disponível em: http://www.planalto.gov.br/, acesso dia: 21/04/2016.

Brasil. Decreto $\mathrm{n}^{\circ}$. 5.296, 02/12/2004. Regulamenta questões associadas à acessibilidade. Disponível em: http://www.planalto.gov.br/, acesso dia: 30/09/2014.

Brasil. Lei no. 13.146, de 06/07/2015. Institui a Lei Brasileira de Inclusão da Pessoa com Deficiência. Disponível em: http://www.planalto.gov.br/, acesso dia: 21/04/2016.

Cavender, Anna; Ladner, Richard E. (2008). Hearing Impairments. Springer, HumanComputer Interaction Series, Web Accessibility, 25-35.

Corradi, Juliane Adne Mesa. (2007). “Ambientes Informacionais Digitais e Usuários Surdos: Questões de Acessibilidade". Dissertação de Mestrado em Ciência da Informação, UNESP, Campus de Marília/SP.

Flor, Carla da Silva; Vanzin, Tarcisio; Ulbricht, Vânia. (2013). Recomendações da WCAG 2.0 (2008) e a Acessibilidade de Surdos em Conteúdos da Web. Rev. Bras. Ed. Esp., Marília, v. 19, n.2, p. 161-168, Abr.-Jun.

ISO/IEC 25010. (2011). "Systems and software engineering. Systems and software Quality Requirements and Evaluation (SQuaRE)". System and software quality models. Disponível em: http://www.iso.org/, acesso dia: 25/07/2011.

Medeiros, Letícia P.; Elia, Marcos; Santos, Mônica P. dos. (2013). "Estratégias para auxiliar o Processo de Aprendizagem da Leitura e Escrita de Alunos Surdos". Em: Anais do XIX Workshop de Informática na Escola (WIE 2013), CBIE.

Ribeiro, Ana Elisa; Fonseca, Gabriel Machado de C.; Santos, Élerson R. S. (2010). "O Site da Escola como Operador do Letramento Digital". Em: Anais do XIX Workshop de Informática na Escola (WIE 2010), CSBC.

Schelp, Patrícia Paula. (2009). "Letramento e Alunos Surdos: Práticas Pedagógicas em Escola Inclusiva”. Em: Anais do IX Congresso Nacional de Educação (EDUCERE), III Encontro Sul Brasileiro de Psicopedagogia.

Silva, Luciano; Bellon, Olga R.P.; Ferreira, Thiago S.; Azevedo, Mauricley R.; Vieira, Fabio L. (2005). "Projeto VisionLinux: integração de ferramentas de acessibilidade para usuários com necessidades especiais". Em: Anais do XI Workshop de Informática na Escola (WIE), XXV CSBC, São Leopoldo/RS.

WAI/W3C. "Web Accessibility Initiative". Disponível em: https://www.w3.org/WAI/, acesso dia 28/11/2014.

WCAG/W3C 2.0. (2008). "Web Content Accessibility Guidelines 2.0”. Disponível em: http://www.w3.org/Translations/WCAG20-pt-br/, acesso dia: 18/11/2014.

WebAIM/AD. (2013). "Web Accessibility in Mind/ Auditory Disabilities: Deaf Culture". Disponível em: http://webaim.org/articles/auditory/culture, acesso dia: 28/11/2014. 